\title{
Unconventional electromagnetic properties of the graphene quantum dots
}

\author{
S. E. Shafraniuk \\ Tegri LLC, Evanston, IL 60202
}

\begin{abstract}
Quantum dots based on the graphene stripes show unconventional optical properties in the $\mathrm{THz}$ frequency range. The graphene quantum dot (GQD) is made of electrically gated stripe with zigzag edges. Inside the active region (AR), which is enclosed between the source and drain electrodes, there are two sharp energy $( \pm)$-levels, whose separation $2 \Delta$ is controlled with Stark effect by applying the lateral dc electric field. Such the edge states determine the unique nature of elementary excitations, chiral fermions, that are responsible for the non-linear optical responce revealing a potential for many applications. They are, e.g., the frequency multiplication and self-focusing of two dimensional solitons. Furthemore, when injection of the non-equilibrium electrons causes an inverse population of the levels localized in AR, the subsequent recombination of electrons and holes leads to a coherent emission of the $\mathrm{THz}$ waves.
\end{abstract}

PACS numbers: DOI: 10.1109

Unconventional optical properties of graphene in the $\mathrm{THz}$ range with frequencies $f=0.5-100 \mathrm{THz}$ attract significant attention of many researchers [1 7]. Interest to the $\mathrm{THz}$ waves (T-rays) is motivated by variety of potential applications in medicine, information technology, communication and security. One example of the T-ray application is the remote sensing of chemical and biological substances that requires powerful $\mathrm{THz}$ lasers and high-resolution spectral analyzers. There are also suggestions of quatum dot $\mathrm{THz}$ detectors [5, 6], frequency multipliers [11 13] and self-focusing of two dimensional solitons 14] in the electrically tunable metastructures. Recently reported [5, 6] carbon nanotube $\mathrm{THz}$ receivers and spectral analyzers exploit the field-induced singleelectron tunneling and transitions between the quantized electron levels. Furthermore, unique plasmonic characteristics of graphene allow building the tunable $\mathrm{THz}$ lasers [7]. The surface plasmons (SP), whose spectrum changes versus the applied gate voltage have been observed in several experiments [15-25] on graphene.

One remarkable feature of graphene is that the carrier concentration, the electrochemical potential $\mu$, and hence its conductivity $\sigma$ can be appropriately tuned, e.g., by applying appropriate electric potentials to the gate electrodes [15, 27 29]. Hence, optical properties of the 2D atomic monolayers are readily tunable in the terahertz $(\mathrm{THz})$ spectral region [15, 30], enabling their application in the compact electrically controllable $\mathrm{THz}$ optical devices [31]. These opens new opportunities as compared to the noble metals that are typically used in $\mathrm{THz}$ devices [27]. Hence, the SP spectrum in the $2 \mathrm{D}$ atomic monolayers is altered in situ, without any changes in the device's design while optical and plasmonic characteristics of the $2 \mathrm{D}$ materials are tunable in the terahertz $(\mathrm{THz})$ spectral region [15, 30].

Currently, there are several concepts of the THz emitters made of the carbon nanotube and graphene [15 18]. A lot of attention is paid to the solid state laser involving localized quantum states arising in systems with reduced dimensionality, e.g., 2D (quantum wells) or 1D (quantum dots). One example are the quantum cascade lasers (QCL) based on layered semiconducting superlattices where series of quantum wells with $2 \mathrm{D}$ electron spectrum are created. A "proof of concept" tunable $\mathrm{THz}$ laser based on the gain modulation by graphene plasmons in an aperiodic lattice and exploiting the unique properties of graphene plasmons was built and tested in Ref. [7]. However, despite their remarkable performance, such the quantum cascade lasers (QCL) have serious setbacks. Basic problem is that the energy dissipation caused by electron-phonon and electron-electron collisions leads to considerable intrinsic Joule heating raising the internal local temperature far above the temperature of the external environment. Such the local Joule heating causes an adverse negative effect on the QCL performance. To reduce the negative effect of the local heating, one should decrease the bias current below some threshold value and also cool down the QCL structure below $200 \mathrm{~K}$. The above measures complicate the QCL design and limit the QCL system power and efficiency. Also there are several concepts of the $\mathrm{THz}$ emitters made of the carbon nanotube and graphene [15, 16]. A "proof of concept" tunable $\mathrm{THz}$ laser based on the gain modulation by graphene plasmons in an aperiodic lattice and exploiting the unique properties of graphene plasmons was built and tested in Ref. [7].

A possible fundamental solution to the above issue of overheating represent systems with lowered dimensionality, e.g., with 1D or even 0D instead of 2D. The electron bands in the 1D and 0D systems are much narrower than in the $2 \mathrm{D}$ systems, which also means that the phase space where the electron-phonon scattering occurs is reduced and most of the electron-phonon scattering processes are eliminated. Hence, in the 1D and 0D systems, the intrinsic energy dissipation due to suppression of the electron phonon scattering is considerably lowered as well. This motivates the interest to electromagnetic properties of low-dimensional comprising quantum dots. Promising examples are the novel 2D atomic monolayers like graphene and its allotropes. The graphene stripes and carbon nanotubes represent 1D systems, whose dimensionality is reduced further to $0 \mathrm{D}$ by introducing an 


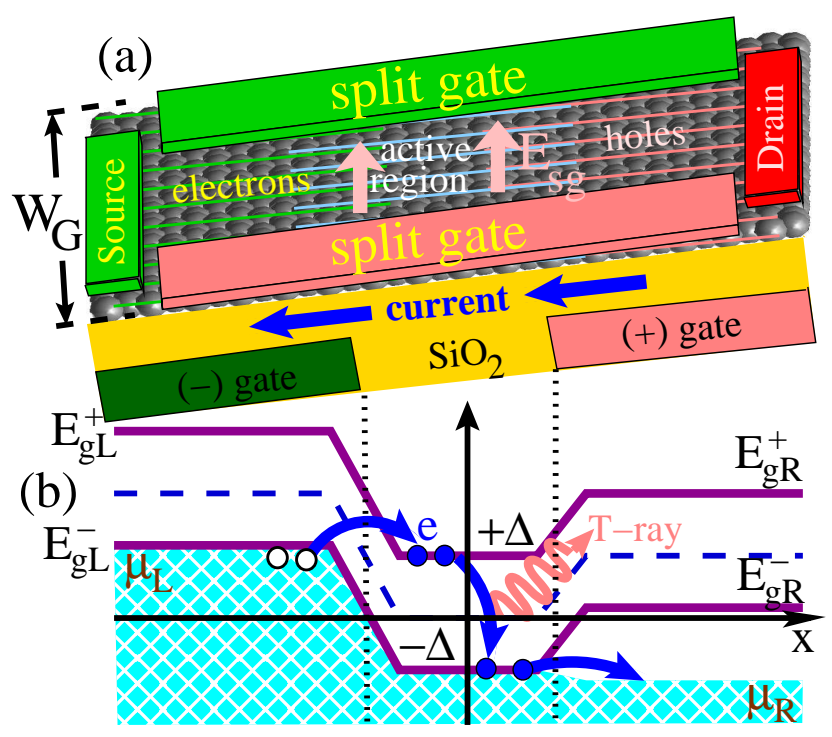

Figure 1: Color online. (a) Geometry of the all-electrically controlled quantum dot (GQD) made of the graphene stripe with zigzag edges. The split gates form a transversal electric field $\mathbf{E}_{\mathrm{sg}}$, which due to Stark effect splits the energy 0-level into the upper (with energy $+\Delta$ ) and lower [with energy $-\Delta$, see sharp peaks in the electron density of states in Fig. $2 \mathrm{~d}$ that originate from the $( \pm)$-levels], whose inverse population is created when a finite bias $V_{S D} \geq 2 \Delta$ is applied between the source and drain electrodes provided that the electron electrochemical potentials $\mu_{L, R}$ in the left and right banks of the graphene stripe are set respectively as $\mu_{L}=E_{\mathrm{gL}}^{-}$and $\mu_{R} \leq E_{\mathrm{gR}}^{-}-\Delta$ using the back $( \pm)$-gates. (b) Energy diagram of GQD.

additional confinement by placing electrodes and local gates. Basically, quantum dot QD lasers are good candidates for the next generation high-speed communication and already are more promising than quantum well lasers with respect to important features like threshold current, temperature stability, chirp, and feedback insensitivity [8-10]. However, there is a need to understand what limits the performance and how it can be improved. This requires a better understanding of the underlying dynamics on a microscopic level. Below we study a graphene quantum dot using microscopic approach for calculating the optical susceptibility.

In this work we consider an all-electrically controlled 0D quantum dot based on the graphene stripe with zigzag edges [32] that comprises a plasmonic THz microcavity. The motivation for this work is the recent success in synthesis of graphene stripes with perfect zigzag edges [33] where according to Refs. 34 37] the topologically protected, sharp and voltage-controlled the edge energy levels emerge. Below we will see, that exploiting such the stable, voltage-controlled edge energy levels opens new

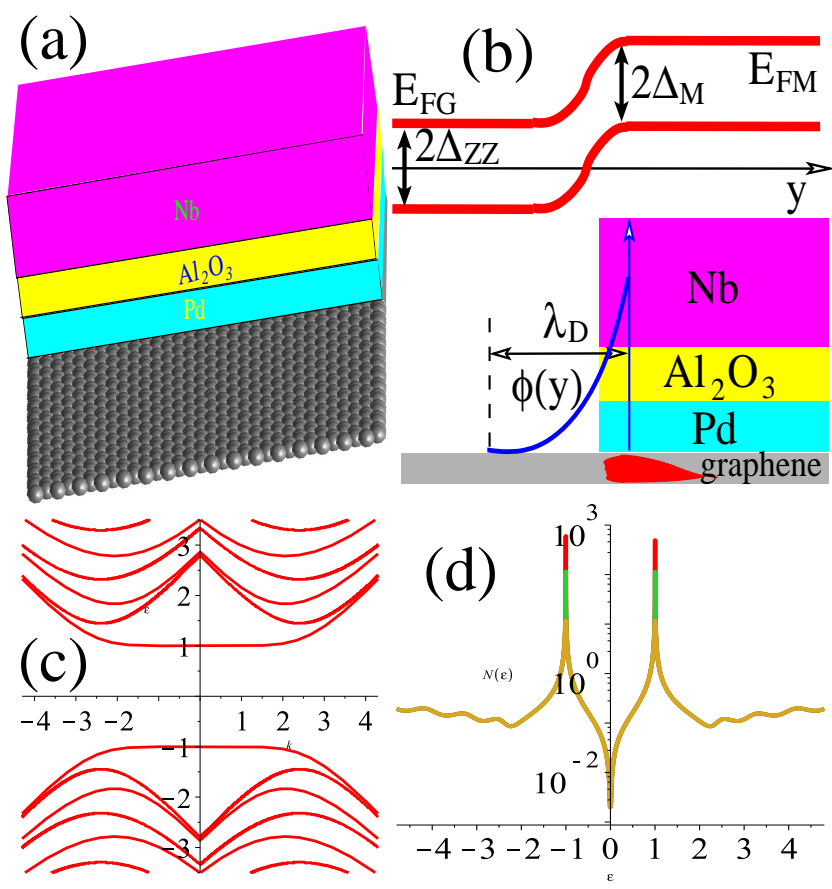

Figure 2: Color online. (a) One side of the $\mathrm{Nb} / \mathrm{Al}_{2} \mathrm{O}_{3} / \mathrm{Pd} / \mathrm{G}$ gate which is deposited on the graphene stripe to form the GQD active region shown in Fig. 1. (b) The energy diagram at the graphene/gate boundary. (c) Electron spectrum showing the subband structure of GQD made of the graphene stripe enclosed between two timber-like split gate electrodes to form the active region with effective zigzag edges shown in Fig. 1]. (d) The local electron density of states $D(\varepsilon)$ inside the GQD active region. The transversal dc electric field $\mathbf{E}_{\mathrm{sg}}$ due to the Stark effect splits the zero-energy peak into two separate peaks forming an energy gap $2 \Delta$.

opportunities for designing the tunable $\mathrm{THz}$ devices. We utilize the unique intrinsic properties of graphene that allow for building various devices with novel remarkable properties. The study is focused on the ability of graphene quantum dot (GQD) to dynamically modulate round-trip modal gain values and shows potential to forming the laser emission. Such the gated 2D monolayer material serves as a powerful tool to controlling the optical properties of GQD. The GQD device is instantly tunable and is all electrical in nature, with minimal electrical power demands.

The goal of this work is to compute the optical susceptibility of the graphene quantum dots (GQD) that describe their unconventional electromagnetic (EM) properties. The efforts are focused on the all-electrically controlled GQD fabricated using the graphene stripes with atomic zigzag edges. The knowledge of how the optical susceptibility of GQD depends on the frequency and electrochemical potential allows better understanding the physical mechanisms related to the electrically controlled absorption and emission of the electromagnetic field. Furthermore, the computation results allow finding, e.g., the conditions to the $\mathrm{THz}$ waves emission by the 
all-electrically controlled GQD. Furthermore our study also focuses on finding the non-linear electromagnetic response of GQD. We will see that the physical mechanism of such the non-linearity originates from the unconventional properties of chiral fermions in graphene stripes with atomic zigzag edges.

\section{THE MODEL}

Geometry of the proposed device is shown in Fig. 19 where the central part is the graphene stripe, whose properties are controlled by the source drain and gate electrodes. The active region represents the graphene quantum dot containing two sharp $( \pm)$-levels originating from the edge states and spaced by $2 \Delta$. Since the edge states are topologically protected [36], the $( \pm)$-levels are very sharp and robust, even if the edge roughness and impurities are present. The magnitude of the level spacing $2 \Delta$ is controlled using the Stark effect by setting the electric voltage between the split gates 37]. Besides, the energy level positions $E_{n}$ in the left (L) and right (R) side sections of the graphene stripe are controlled by applying electric potentials $V_{\mathrm{GL}, \mathrm{R}}$ to the left and right side bottom gate electrodes respectively. Furthermore, by applying a finite bias voltage between the source and drain electrodes one injects the non-equilibrium electrons into the upper level with energy $E_{+}=+\Delta$, thereby creating an inverse population of the upper $(+)$-level in the active region. The electrons residing on the upper $(+)$ level then recombine to the lower $(-)$ level by emitting $\mathrm{THz}$ photons. Hence, the subsequent recombination of the electrons into the lower level with $E_{-}=-\Delta$ leads to a emission of the T-rays with frequency $f=2 \Delta / h$. The interaction between light and material is controlled by the shape of the electromagnetic density-of-states (DOS) in the micro resonator [38, 39]. These mean that the magnitudes of lasing frequency $f$ and the amplification of resonant modes are set by applying the split-gate voltage $V_{\mathrm{SG}}$ across the active region of the laser and/or by the source drain and bottom gate voltages as shown in Fig. 1. The latter mechanism is studied in details below in Sec. V. We will see that the resonant frequency of $\mathrm{THz}$ emission depends not only on the split gate voltage $V_{\mathrm{SG}}$ as mentioned above but also it varies versus the electrochemical potential $\mu$, which is controlled by applying voltage to the gate electrodes. This enables flexible all-electric manipulations the lasing emission parameters [40 42].

Understanding the mechanisms determining the optical properties of GQD is accomplished using solutions the Dirac equation complemented by appropriate boundary conditions (see Refs. [4, 32, 34 37] for details).

\section{SUSCEPTIBILITY OF GRAPHENE}

Initially we consider a simplest case of the twodimensional (2D) electron gas in the atomic monolayer representing a suspended pristine graphene. A general expression for the optical susceptibility of free carriers in graphene that are chiral fermions (HF) takes the form

$$
\chi(\mathbf{q}, \omega)=\frac{\left|d_{c v}\right|^{2}}{L^{2}} \sum_{s, s^{\prime}= \pm 1} \int \frac{d^{2} \mathbf{k}}{(2 \pi)^{2}} \frac{f\left(\epsilon_{s, \mathbf{k}+\mathbf{q}}\right)-f\left(\epsilon_{s^{\prime}, \mathbf{k}}\right)}{\omega-\epsilon_{s, \mathbf{k}+\mathbf{q}}+\epsilon_{s^{\prime}, \mathbf{k}}+i \eta}
$$

where $d_{c v}$ is the electric dipole matrix element, whose indices $c, v$ are attributed to the conducting/valence bands, $L$ is the size of a square-shaped 2D sample, $\eta$ is the damping parameter associated with the electron energy dissipation during the inelastic collisions, $\mathbf{k}$ is the $2 \mathrm{D}$ electron momentum, $\mathbf{q}$ and $\omega$ are the respective electron momentum and energy change, $f\left(\epsilon_{s, \mathbf{k}}\right)$ is the HF distribution function that depends on the HF excitation energy $\epsilon_{s, \mathbf{q}}$, which for the pristine graphene conform the continous dispesion law

$$
\epsilon_{s, \mathbf{q}}=s v_{F}|\mathbf{q}|
$$

where $s$ and $s^{\prime}= \pm 1$ are the HF branch indices, $v_{F}$ is the Fermi velocity in graphene. The damping parameter $\eta$ in Eq. (1) actually plays the same role as parameter of the adiabatic switch-on. For the HF spectrum (2), the density of states is 32]

$$
D_{G}(\epsilon)=\frac{3 \sqrt{3} a^{2}}{\pi v_{\mathrm{F}}^{2}}|\epsilon|
$$

where $a$ is the lattice constant in graphene.

The calculation details of the optical susceptibility are given in Appendix A. Results of the calculation are illustrated in Figs. 3 where we show the real $\chi^{\prime}=\Re \chi$ and imaginary $\chi^{\prime \prime}=\Im \chi$ parts of the optical susceptibility versus the frequency $\omega$ and electrochemical potential $\mu$. As an illustaration, we also show results for a conventional semiconductor with dimensionalities $D=1,2,3$ (see Fig. 3a,b). Parameters of the calculations, whose details are given in Appendix A, are indicated in the caption. Respective results for $\Re \chi(\omega)$ (curve 1) and $\Im \chi(\omega)$ (curve 2) of the pristine graphene are shown in Fig. 3 . while Fig. 3 illustrates results for $\Re \chi(\mu)$ (curve 1) and $\Im \chi(\mu)$ (curve 2). Figure Fig. Be shows the results for $\chi$ versus the charge carrier concentration $n$, as indicated in the caption. The last Fig. 3. shows dependence of the HF charge carrier concentration $n$ versus the electrochemical potential $\mu$ for pristine graphene.

At finite temperatures and in the non-equilibrium conditions, the respective calculations of $\chi(\omega, \mu)$ are conducted numerically. Numeric solutions are also useful when considering graphene-based structures with more complicated excitation spectrum. Below we study the optical properties of the graphene stripe with zigzag edges and non-linear optical properties of GQD when a strong ac field is present. 

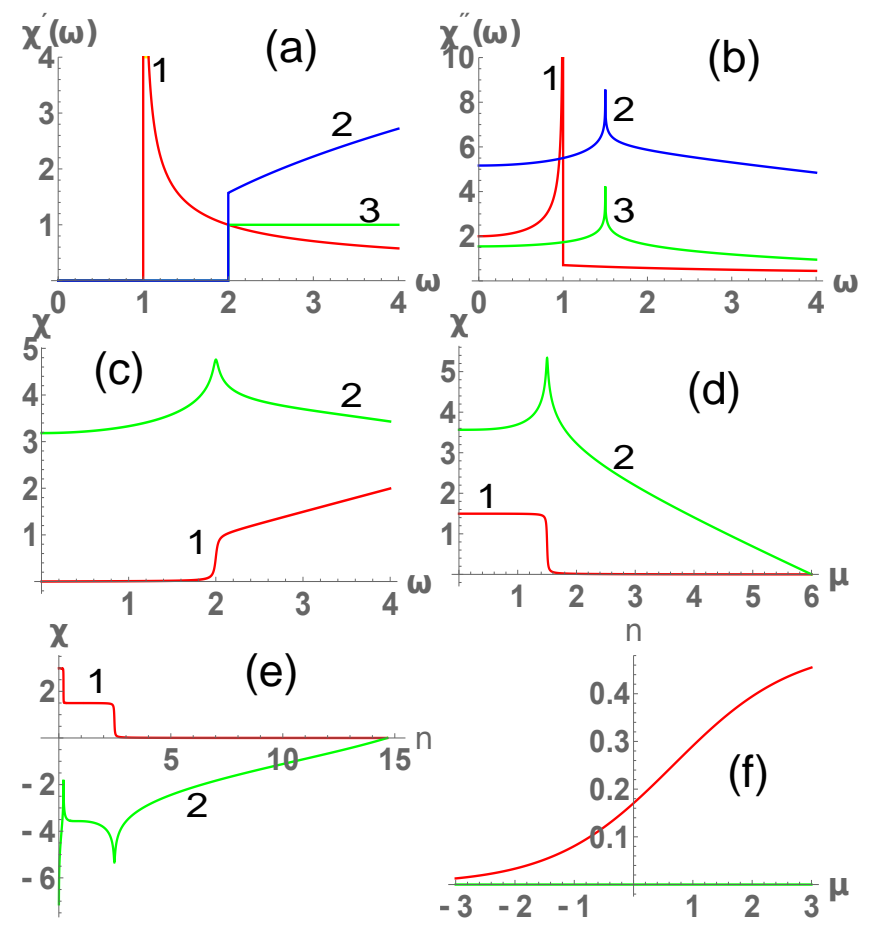

Figure 3: Color online. Optical susceptibility: (a) $\Re \chi(\omega)$ for $D=1 . .3$ (respective curve labels are 1,2 and 3 ), $\delta=1$, $\mu=0.5$, and $\Lambda=36$. (b) $\Im \chi(\omega)$ for $D=1 . .3$ (respective curve labels are 1,2 and 3 ), $\delta=1, \mu=0.5$, and $\Lambda=16$. (c) and (d) The real (curve 1) and imaginery (curve 2) parts of the pristine graphene susceptibility $\chi(\omega)$ (see (c) for $\mu=1$ ) and $\chi(\mu)$ (see (d) for $\omega=3$ ) respectively versus the frequency $\omega$ and electrochemical potential $\mu$ for $\eta=0.02, \Lambda=6$. (e) The real (curve 1) and imaginary (curve 2) $\chi(n)$ for the pristine graphene for $\eta=0.2, \Lambda=6, \mu=1$. (f) The HF excitations concentration $n(\mu)$ versus the electrochemical potential $\mu$ for pristine graphene.

\section{ZZ STRIPE OF GRAPHENE}

For graphene stripes, whose width $W$ is finite, one should also consider quantization of the HF excitations in the lateral direction. Below we compute optical susceptibility of the graphene stripe with atomic zigzag edges. We use units with $\hbar=1$ and $k_{\mathrm{B}}=1$ if not stated otherwise. The edge states [4, 32, 34-37] emerging in the graphene stripe change the $\mathrm{HF}$ excitation spectrum significantly. This happens because the chirality of excitations in graphene imposes additional constrains on reflection processes due to conservation of two pseudospins. A transverse d.c. electric field $\mathbf{E}=\left\{0, E_{y}, 0\right\}$ is applied perpendicular to the graphene stripe axis utilizing the split gate electrodes shown in Fig. 17. When $E_{y}=0$, the electron reflections at the atomic zigzag edges [34, 35, 37] cause a crisp narrow energy level to arise at the energy $\epsilon=0$. When the transverse electric field is finite, $E_{y} \neq 0$, such the zero-energy level experiences Stark splitting, whose magnitude is $2 \Delta=2 e E_{y} W$ (see Refs. [4, 32, 36, 37]). Then, the Stark splitting of the sharp singularities at energies $\epsilon= \pm \Delta$ emerging in the electron density of states (see Fig. 2 $\mathrm{d}$ ) is controlled by the electric field $E_{y} \neq 0$. Below we will see that such the electrically controlled HF spectral singularities are responsible for the remarkable optical properties of the graphene quantum dots. The effect is described in terms of the susceptibility $\chi(\omega, \mu)$, which we compute below. An additional control of $\chi(\omega, \mu)$ is introduced with the top (or bottom) local gate electrodes (see Fig. 1a). Thus, the respective local gate voltages control the both, the Stark splitting $\Delta$ along with the HF electrochemical potential $\mu$. Below we will see that the shape of $\chi(\omega, \mu)$ depends on both, $\mu$ as well as on $\Delta$.

There is no simple analytical expression for the HF excitation energy in the graphene stripe with atomic zigzag edges [4, 32, 34 37]. The HF dispersion law [4, 32, 34 37] is given by the two equations

$$
\begin{gathered}
\epsilon_{k}=\mu \pm \sqrt{\Delta^{2}+k_{y}^{2} v_{\mathrm{F}}^{2}+k_{x}^{2} v_{\mathrm{F}}^{2}} \\
k_{y}=\frac{k_{x}}{\tan \left(W k_{x}\right)}
\end{gathered}
$$

that describe the constrain due to the pseudospin conservation during elastic reflections of the $\mathrm{HF}$ excitations on atomic edges. The electron energy in graphene stripe (4) depends on the two components of the electron (HF) momentum, $k_{y}$ (longitudinal) and $k_{y}$ (transversal), which are related by Eq. (5). In an infinitely long stripe by width $W, k_{y}$ is continuous while $k_{x}$ discrete, because the transversal momentum is quantized. In the geometry on Fig. 1a, the active region length $L_{a}$ is $L_{a}>>W$, therefore the quantization along the $y$-axis is negligible. We will see that such the constrain allows reducing the dimensionality of the system from $2 \mathrm{D}$ as in pristine graphene to $1 \mathrm{D}$ for the stripe. In Eqs. (4), (5), $\epsilon_{k}$ is the $\mathrm{HF}$ energy variable, $\mu$ is the electrochemical potential, $\Delta=e E_{y} W$ is the Stark splitting of the zero-energy level posing as the "energy gap" in Eq. (44). In such the case, the splitting energy also depends on the gate efficiency $\alpha$. Magnitude of $\mu$ is controlled by the bottom (or top) gate electrodes, while $\Delta$ is controlled by the split gate electrodes depicted in Fig. 17a as illustrated by energy diagram in Fig. 1 b. The HF dispersion law is computed by solving Eqs. (4), (5). The HF excitation energy $\epsilon\left(k_{y}\right)$ in the graphene stripe with zigzag edges is shown in Fig. 2r versus the longitudinal momentum $k_{y}$. Technically, the electron density of states shown in Fig. $2 \mathrm{~d}$ is computed as

$$
D_{\mathrm{ZZ}}(\epsilon)=\left|\frac{d k_{y}\left(k_{x}\right)}{d \epsilon_{k}}\right|=\left|\frac{d k_{y}\left(k_{x}\right)}{d k_{x}} / \frac{d \epsilon_{k}}{d k_{x}}\right|
$$

which gives an analytical expression

$$
D_{\mathrm{ZZ}}(\epsilon)=\left|\frac{\epsilon_{k}}{k_{x}} \frac{\tan k_{x} W-k_{x} W\left(\tan ^{2} k_{x} W+1\right)}{\tan ^{2}\left(k_{x} W\right)}\right|
$$


where according Eqs. (4), (5), $k_{x}$ depends on the energy variable $\epsilon$. To compute $\chi(\omega, \mu)$ for the quasi-1D graphene stripe with atomic zigzag edges, we again use the general expression Eq. (1). The calculation is simplified for the direct interband transitions $(\mathbf{q}=0)$. Then we get

$$
\begin{aligned}
\chi(\omega)= & \frac{\left|d_{c v}\right|^{2}}{2 \pi a^{2}} \int \frac{\left[f\left(\epsilon_{k}-\mu\right)+f\left(\epsilon_{k}+\mu\right)-1\right]}{\omega \pm 2 \epsilon_{k}+i \eta} d k_{y} d k_{x} \\
= & \frac{\left|d_{c v}\right|^{2}}{2 \pi a^{2}} \int_{k_{\max }}^{k_{\min }} D_{\mathrm{ZZ}}\left(k_{x}\right)\left[1-f\left(\epsilon_{k}-\mu\right)-f\left(\epsilon_{k}+\mu\right)\right] \\
& \times\left(\frac{1}{\omega-2 \epsilon_{k}+i \eta}-\frac{1}{\omega+2 \epsilon_{k}+i \eta}\right) d k_{x}
\end{aligned}
$$

where we use

$$
\begin{array}{r}
f\left(\epsilon_{s, k}\right)-f\left(\epsilon_{s^{\prime}, k}\right) \\
=f\left(-\mu+\epsilon_{k}\right)-f\left(-\mu-\epsilon_{k}\right)= \\
\pm\left[f\left(\epsilon_{k}-\mu\right)+f\left(\epsilon_{k}+\mu\right)-1\right]
\end{array}
$$

and

$$
d k_{y}=D_{\mathrm{ZZ}}\left(k_{x}\right) d k_{x}
$$

When integrating (8) in infinite limits, the respective integral diverges. Therefore we introduce cutoff by setting the lower $k_{\min }$ and upper $k_{\max }$ limits of integration in Eq. (8), which respectively are found as solutions of the equations

$$
k_{\min }^{2}=\mu^{2} / v_{\mathrm{F}}^{2}-\left[k_{y}\left(k_{\min }\right)\right]^{2}-\left(\Delta / v_{\mathrm{F}}\right)^{2}
$$

and

$$
k_{\max }^{2}=\Lambda^{2} / v_{\mathrm{F}}^{2}-\left[k_{y}\left(k_{\max }\right)\right]^{2}-\left(\Delta / v_{\mathrm{F}}\right)^{2} .
$$

Above we have used that the respective change of the $\mathrm{HF}$ excitation energy is $-\epsilon_{s, k}+\epsilon_{s^{\prime}, k}= \pm 2 \epsilon_{k}$, where $\epsilon_{\kappa}$ is defined by Eq. (4). The above Eqs. (5), (11) and (12) serve as the closed system of transcendental equations allowing to finding $k_{\min }$ and $k_{\max }$. From Eq. (6) one can see that in contrast to pristine graphene, whose the HF density of states (3) is a smooth function of the energy variable $E$, the respective density of states $D_{\mathrm{ZZ}}(\epsilon)$ given by Eq. (6) for the graphene stripe with zigzag (ZZ) edges shows dramatically different behavior, as illustrated in Fig. 2 d. Namely, owing to appearance of ZZ edge states in the graphene stripe, the respective singularities in the density of states (6) arise when $\tan ^{2}\left(k_{x} W\right)=0$ in denominator of Eq. (7) provided

$$
k_{x}=\frac{\pi}{W} m
$$

where $m$ is integer. One can notice the mentioned sharp singularities at energies $\epsilon= \pm \Delta$ (in units of Stark splitting $\Delta$ ) in the plot of DOS on Fig. [2d., while the singularities are smoothed at energies, $\epsilon>\Delta$ and $\epsilon<-\Delta$. The number of excitations in the ZZ graphene stripe is computed as

$$
N=2 \sum_{\mathbf{k}} f_{k} \rightarrow \frac{12 \sqrt{3} a^{2}}{\pi v_{F}^{2}} \int_{k_{\max }}^{k_{\min }} D_{\mathrm{ZZ}}\left(k_{x}\right) f\left(\epsilon_{k}-\mu\right) d k_{x}
$$

where the lower and upper integration limits are again determined by solution of Eqs. (5), (11) and (12).

The relevant energy scale in the above formulas (8)(14) is determined by the atomic edge geometry and by the graphene stripe width $W$. Other energy scales in Eqs. (8) -(14) are related to $\omega, \Delta, \mu$ and $\eta$. Typical magnitudes of interest here are $\omega \sim 2 \pi f$, where for the electromagnetic field frequency $f=1 \mathrm{THz}$, the respective photon energy is $h f=4 \mathrm{meV}$. Then, $\mu \sim 2 \Delta \sim h f=4 \mathrm{meV}$, and we also use $\eta \sim 0.1 \Delta$. Essentially, the last parameter, $\eta$, which also determines the width of quantized levels localized in the active region depends on coupling of GQD to the substrate and also by the inelastic collisions, which also depend on the temperature and GQD geometry.

\section{ELECTROMAGNETIC EMISSION FROM GQD}

A fundamental problem when designing the laser for the frequency region $0.5-100 \mathrm{THz}$ is that the $\mathrm{THz}$ photon energy $E_{\nu}^{\mathrm{T}}$ is relatively low, $E_{\nu}^{\mathrm{T}} \approx 4 \mathrm{meV}-0.4 \mathrm{eV}$, as compared to a visible light photon for which $E_{\nu}^{\mathrm{L}} \approx 1.8$ - $3 \mathrm{eV}$. Therefore, to ensure a monochromatic THz emission, the width $\eta$ of quantized levels localized in the active region is required to be much narrower than $\Delta$. This problem is solved by a proper designing the active region, which is the key element of any solid state laser. Parameters of the active region must satisfy to a number of requirements, which have to be observed in order to get the T-ray emission out of it. In conventional visible light lasers, $\eta$ is typically much smaller than the level spacing between the e/h-levels, i.e., $\eta<<\Delta$. Then, the photon energy $E_{\nu}^{\mathrm{L}}$ is precisely equal to the level spacing energy $\Delta$, i.e., $E_{\nu}^{\mathrm{L}} \equiv \Delta$ while the emitted light beam is fairly monochromatic and coherent. The situation is different in the $\mathrm{THz}$ lasers where at the bottom part of the $\mathrm{THz}$ domain the condition $\eta<<\Delta$ might fail if the level broadening $\eta$ exceeds $\Delta$, which is relatively small, $\Delta \approx 4 \mathrm{meV}$. In the latter case, the spectrum of photon emission acquires the finite width (line broadening) while the photon energy distribution becomes dependent also on the width $\eta$ of the electron energy level $E_{\nu}$. Then the finite $\eta$ causes an extra decoherence and broadening the $\mathrm{THz}$ laser emission spectrum. In a worst case scenario one can even get $\eta \geq \Delta$, which causes complete violating of the condition $E_{\nu}^{\mathrm{T}} \equiv \Delta$. The latter example illustrates why creating the $\mathrm{THz}$ lasers is so difficult. Other sources the T-beam decoherence and the line broadening consist of the temperature fluctuations and noises which also strongly impact the $\mathrm{THz}$ device performance. Using of graphene suggests several possible solutions of the mentioned decoherence problem. (i) One is able to form very narrow electron energy levels in the active region of the $\mathrm{THz}$ laser where $\eta<<\Delta$. Very sharp and narrow e/h-levels are obtained inside a narrow stripe of graphene with zigzag (ZZ) edges polarized by a transverse electric field as suggested in Refs. [4, 32, 34 37]. (ii) One can 
significantly reduce impact of phonons which essentially contribute into the level width $\eta$. It is accomplished when using of a narrow stripe having a definite orientation in respect to crystallographic directions of the graphene lattice. The lattice symmetry and the atomic edges impose additional selection rules on the scattering probability involving just phonons with certain polarization and wave vector on the one hand and electrons with onedimensional dispersion law on the other hand. Such the restriction rules out many inelastic scattering processes as irrelevant. Furthermore, due to narrow width $\delta$ of the electron bands in GQD, the high-energy phonons with frequency $f_{p h}>\delta / h$ do not participate in the electronphonon scattering as well. The other mechanism involving the electron-electron collisions is less significant for graphene in the $\mathrm{THz}$ frequency range. The above results in very low energy dissipation in the graphene stripes with zigzag edges. (iii) The levels originate from the topologically protected edge states [36], hence they are robust in respect to the lattice imperfections and thermal excitations.

Here we consider the limit of high density of the charge carriers, whose energy recombination time $\tau_{\mathrm{HF}}$ is much shorter than the change in polarization of the electromagnetic wave. The polarization relaxes very fast, and is governed by the carrier-carrier and carrier-phonon scattering causing the relaxation to its quasi-equilibrium value, which is determined by the momentary magnitudes of the field and the carrier density. This ensures the simplest quasi-equilibrium conditions of a stationary excitation when the carriers are in equilibrium with themselves while the graphene stripe is out of equilibrium. In the quasi-equilibrium approximation, the field intensity is a slow functions of time. We disregard all the effects causing deviations from the quasi-equilibrium assumption, such as spectral, spatial, or kinetic hole burning. This enables using the electron-hole-pair rate equation complemented with laser specific terms. The rate equation for generating of $N$ photons takes the form

$$
\dot{N}=r_{p}-r_{s t}-r_{s p}-r_{n r},
$$

where $r_{p}$ is the pumping rate, $r_{s t}$ is the stimulated emission rate, $r_{s p}$ is the spontaneous emission rate, $r_{n r}$ is the non-radiative transitions rate. The pump rate due to injection current density $j$ is $r_{p}=j \eta_{Q E} /(\mathrm{eW})$ where $\eta_{Q E}$ is the quantum efficiency, $W$ is the transverse dimensions of the laser's active region (i.e., the stripe width). The stimulated emission loss rate is $r_{s t}=$ $-\chi^{\prime \prime}(\omega) \mathcal{E}_{0}^{2} /(2 \hbar)$ where $\omega=2 \pi f$. The quasi-equilibrium susceptibility $\chi(\omega)$ contains the factor $1-f_{e, k}-f_{h, k}=$ $\left(1-f_{e, k}\right)\left(1-f_{h, k}\right)-f_{e, k} f_{h, k}$ that is conveniently separated in the two terms as $\chi^{\prime \prime}(\omega)=\chi_{a}^{\prime \prime}(\omega)-\chi_{e}^{\prime \prime}(\omega)$. Here $f_{e(h), k}$ is the distribution function of electron(hole)-like $\mathrm{HF}$ excitations and the term $\chi_{e}^{\prime \prime}(\omega) \propto f_{e, k} f_{h, k}$ describes the emission while the other term $\chi_{a}^{\prime \prime}(\omega)$ describes the absorption, $\chi_{a}^{\prime \prime}(\omega) \propto\left(1-f_{e, k}\right)\left(1-f_{h, k}\right)$. The imaginary part of susceptibility $\chi_{e}^{\prime \prime}(\omega)$ is related to the gain $g(\omega)$ as

$$
g_{e}(\omega)=-\frac{4 \pi}{n_{b} c} \omega \chi_{e}^{\prime \prime}(\omega),
$$

where $g_{e}(\omega)$ is the probability per unit length to emitting a photon and the background refractive index is $n_{b} \simeq \sqrt{\epsilon_{0}}$. Thus $-g_{e} c / n_{b}$ is the emission probability of a photon per unit time. The spontaneous emission rate into the continuum of all photon modes with the frequency $\omega=\omega_{q, \lambda}$ where $q, \lambda$ are the photon wave vector and polarization is

$$
r_{s p}=\frac{4}{\pi \epsilon_{0}} \int d q q^{2} \omega_{q} \chi_{e}^{\prime \prime}\left(\omega_{q}\right)
$$

or

$$
r_{s p}=\frac{1}{2 \pi \epsilon_{0}} \int_{0}^{\infty} d \omega\left(\frac{2 \omega n_{b}}{c}\right)^{3} \chi_{e}^{\prime \prime}(\omega) .
$$

The dependence $\sim \omega^{3}$ indicates that the spontaneous emission losses dominate at higher laser frequencies. The non-radiative emission rate is computed as $r_{n r}=$ $N / \tau+C N^{3}$ where the 1 -st term corresponds to the multiphoton emission involving deep trap levels while the 2-nd term might contain a significant contribution from the Auger processes in the $\mathrm{THz}$ lasers.

The T-ray laser emission is described in terms of the semi-classical electric field equation for spatial eigenmodes

$$
\left[1+4 \pi \chi(N) / \epsilon_{0}\right] \ddot{\mathcal{E}}_{n}+\left(\kappa c / n_{b}\right) \dot{\mathcal{E}}_{n}+\omega_{n}^{2} \mathcal{E}_{n}=0
$$

where where $\omega_{n}$ is the eigenfrequency of the $n$-th resonator mode and we have introduced the cavity loss rate as $\kappa c / n_{b}=4 \pi \sigma / \epsilon_{0}$ where $\sigma$ is the electric conductivity. For Eq. (19), there are two regimes of the steady state solutions: $(i)$ When the gain $g(\omega=0)$ is less than the cavity losses, the laser field vanishes, i.e., for $\kappa>g\left(N_{0}, \omega_{m}\right)$ one gets the magnitude of time - averaged (i.e., at $\omega=0$ ) electric field $\mathcal{E}_{0}=0$ and $r_{p}=N_{0} / \tau$, where $N_{0}$ is the time - averaged number of photons. (ii) If the gain becomes equal or exceeds the cavity losses provided $\kappa=g\left(N_{0}, \omega_{m}\right)$, one gets the finite magnitude of laser field, i.e., $\mathcal{E}_{0} \neq 0$. Namely,

$$
\mathcal{E}_{0}^{2}=\frac{2 \hbar}{\chi^{\prime \prime}\left(N_{0}\right)}\left(\frac{N_{0}}{\tau}-r_{p}\right)
$$

and

$$
\omega_{m}^{2}=\frac{\omega_{n}^{2}}{1+4 \pi \chi^{\prime}\left(N_{0}\right) / \epsilon_{0}},
$$

where $\omega_{m}$ is the lasing frequency and $\chi^{\prime}$ and $\chi^{\prime \prime}$ are the real and imaginary parts of the optical susceptibility in the active region, $\epsilon_{0}$ is the background dielectric constant. The above formula for $\omega_{m}^{2}$ suggests that the pulling of the laser mode is caused by the refractive index changes due to the increased carrier density that is controlled by the gate voltages. 


\section{STEADY STATE SUSCEPTIBILITY OF GQD}

Below we consider the effect of the electrochemical potential $\mu$ on the optical susceptibility of the graphene quantum dot (GQD), which is controlled by applying electric potentials to the back (or top) gate electrodes. The calculation results for the steady state optical susceptibility $\chi(\omega)$ are presented in Figs. 4, 5, 7 and 8 .

The real $\Re \chi(\omega)$ and imaginary $\Im \chi(\omega)$ parts are shown in Fig. 4 as functions of frequency $\omega=2 \pi f$ for different values of the electrochemical potential $\mu$, whose respective values are indicated in the figures. One can see that the frequency dependence of $\chi(\omega)$ dramatically changes as $\mu$ varies. Physically, this reflects the drastic change of the GQD optical properties since the magnitude of $\mu$ determines the quantization conditions at the graphene stripe edges. Remarkably, as $\mu$ changes, the signs and magnitudes of the real and imaginary parts of $\chi(\omega)$ alter. In Fig. 5, we detalize the instability regions in the narrower frequency intervals. One can see that in certain frequency intervals the real part vanishes, $\Re \chi(\omega)=0$, while $\Im \chi(\omega)<0$ remains negative. An important conclusion drawn from Figs. 4 and 5 is that there is a set of resonant frequencies $\Omega_{\mathrm{p}}$ determined by the condition $\left.\Re \chi(\omega)\right|_{\omega=\Omega_{\mathrm{p}}}=0$ provided $\Im \chi(\omega)<0$. Remarkably, the $\Omega_{\mathrm{p}}$ magnitude depends on $\mu$ and $\Delta$, so in experments it can be controlled by applying appropriate electric potentials to the gate electrodes. Hence, based on the data presented in Figs. 4 and 5, one concludes that lasing conditions are fulfilled at $6.93<\omega<7.03,6.96<\omega<7.09$ and $7.14<\omega<7.22$ (in units of $\Delta$ ). However, when $\Im \chi(\omega)$ becomes positive (see peaks of $\Im \chi(\omega)$ in Fig. 5), the lasing condition fails.

At the first sight it seems there is a problem with generating the coherent $\mathrm{THz}$ radiation with the resonant frequency $\Omega_{p} \sim 6 \Delta /$ hbar when $\Omega_{p}<3 \mathrm{THz}$, which requires the minimum level spacing $\Delta<3 \mathrm{THz} / 6=0.5 \mathrm{THz}$. Such the narrow level spacing $\sim 2 \mathrm{meV}$ corresponds to $k_{\mathrm{B}} T \sim 20 \mathrm{~K}$, which is far below the room temperature $T_{\text {room }} \sim 300 \mathrm{~K}$. Deceptively, it seems that at $T=T_{\text {room }} \sim 300 \mathrm{~K}$, the large temperature broadening $\eta \sim 30 \mathrm{meV}$ smears the spectral singularities at $\epsilon= \pm \Delta$ out because it largely exceed the level spacing $2 \Delta=4 \mathrm{meV}$, thereby making the levels non distinguishable. However, according to detailed calculations (see e.g., Ref. [49]) for the narrow levels in the graphene stripes with zigzag edges, the actual level broadening due to the inelastic scattering is two orders of magnitude lower than for conventional electrons with continuous dispersion law. The physical reason is that the localized HF excitations interact with phonons very weakly, because the respective phase space is confined. Therefore, the actual broadening of the $( \pm)$ levels is very low and is below $3 \mathrm{meV}$ even at $T=T_{\text {room }} \sim 300 \mathrm{~K}$. Thus, for narrower separation down to $2 \Delta \sim 3 \mathrm{meV}$ in GQD that corresponds to $f \sim 0.7 \mathrm{THz}$, the levels remain well-defined even at room temperature.

To further illustrate the capability of the graphene
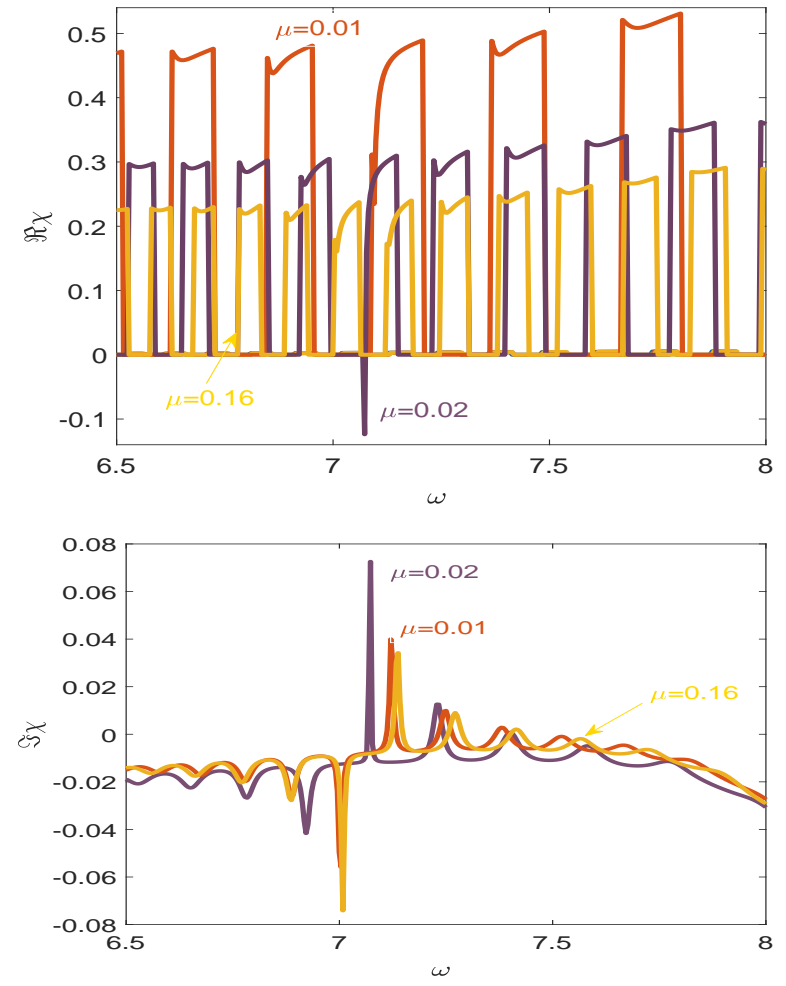

Figure 4: Color online. The steady state optical susceptibility $\Re \chi(\omega)$ (top panel) and $\Im \chi(\omega)$ (bottom panel) as function of frequency $\omega=2 \pi f$ in GQD computed for the stripe width $W=2 h v_{\mathrm{F}} / \Delta$, temperature $T=1.4$ (in units of $\Delta / k_{\mathrm{B}}, k_{\mathrm{B}}$ is Boltzmann constant), the ineleastic collision rate $\eta=0.15$ and the level spacing (Stark splitting) $2 \Delta=2$ (all in units of $\Delta$ ). Here $\omega=2 \pi f, f$ is the frequency and $\mu$ is the electron electrochemical potential. The respective values of $\mu$ are shown in figure.

quantum dot to forming the favorable lasing conditions we plot the dependenlies $\Re\{\chi(\mu)\}$ and $\Im\{\chi(\mu)\}$ on the electrochemical potential $\mu$ in Fig. 6. From Fig. 6] one can see that by changing $\mu$, one alters the shape of the susceptibility curves $\chi(\mu)$ considerably, thereby enabling the flexible control over the coherent T-ray emission. Furthermore, the fast switchings of $\Re\{\chi(\mu)\}$ and $\Im\{\chi(\mu)\}$ takes place when $\mu$ hits the quantized level positions, which have narrow spacing and are dense for the relatively broad stripe $W=2 h v_{\mathrm{F}} / \Delta$. The gain versus frequency is shown in Fig. 7 (top panel). We also present more detailed plot in a narrower frequency region Fig. 7 (bottom panel). From this Fig. 7 one can see that for the listed GQD parameters (i.e., the stripe width $W=2 h v_{\mathrm{F}} / \Delta$, temperature $T=1.4 \Delta / k_{\mathrm{B}}$, the ineleastic collision rate $\eta=0.15 \Delta$ and the Stark splitting $2 \Delta)$, the gain exceeds the cavity loss. Provided $\Re\{\chi(\omega, \mu)\} \sim 0$, which is the case in certain intervals of $\mu$, one achieves the necessary conditions for the coherent T-ray emission.

A general insight into the sign switching of the optical susceptibility for the graphene quantum dot versus $\omega$ and 


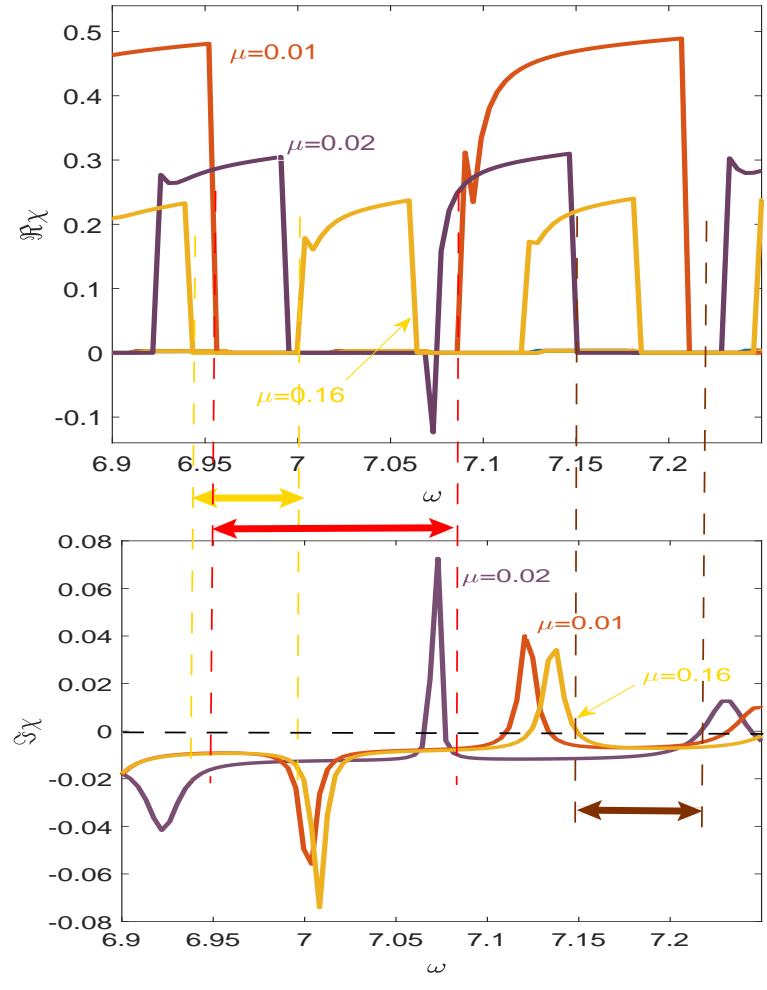

Figure 5: $\quad$ Color online. Blowup $\Re \chi(\omega)$ (top panel) and $\Im \chi(\omega)$ (bottom panel) shown in the former Fig. 4 in the narrower frequency interval. The instability regions corresponding to the conditions $\left.\Re \chi(\omega)\right|_{\omega=\Omega_{\mathrm{p}}}=0$ provided $\Im \chi(\omega)<0$ are marked by arrows (the arrow colors correspond to the curve collors). They correspond to a set of resonant frequencies $\Omega_{\mathrm{p}}$ determined by the mentioned conditions.

$\mu$ is given in the contour plot of $\Im \chi(\omega, \mu)$ as shown in Fig. 8 (top panel) where the green regions correspond to $\Im \chi<0$ while yellow areas to $\Im \chi>0$. Interesting, the sign switch does not happen for much narrower stripes with $W=0.2 h v_{\mathrm{F}} / \Delta$ where the level spacing is wide, although the whole dependence $\Im \chi(\omega, \mu)$ becomes much smoother as shown in Fig. 8 (bottom panel).

When designing the graphene $\mathrm{THz}$ lasers, there are other potential issues as follows. (a) Pumping of nonequilibrium electron and hole excitations into the active region of the $\mathrm{THz}$ laser causes not only inverse population of the e/h-levels. An adverse side effect is that the non-equilibrium electrons and holes eventually transfer their excessive energy to the lattice oscillations and to other excitations in the system. This leads to an overall heating of the active region during the induced emission process. Excessive Joule heating of the active region might change its properties and can even adversely impact the overall performance of the $\mathrm{THz}$ laser. Therefore, one should pay attention to reducing the unwanted heating. The adverse heating can be diminished by implementing the active region with an appropriate geometry, crystallographic orientation, and dimensions. In
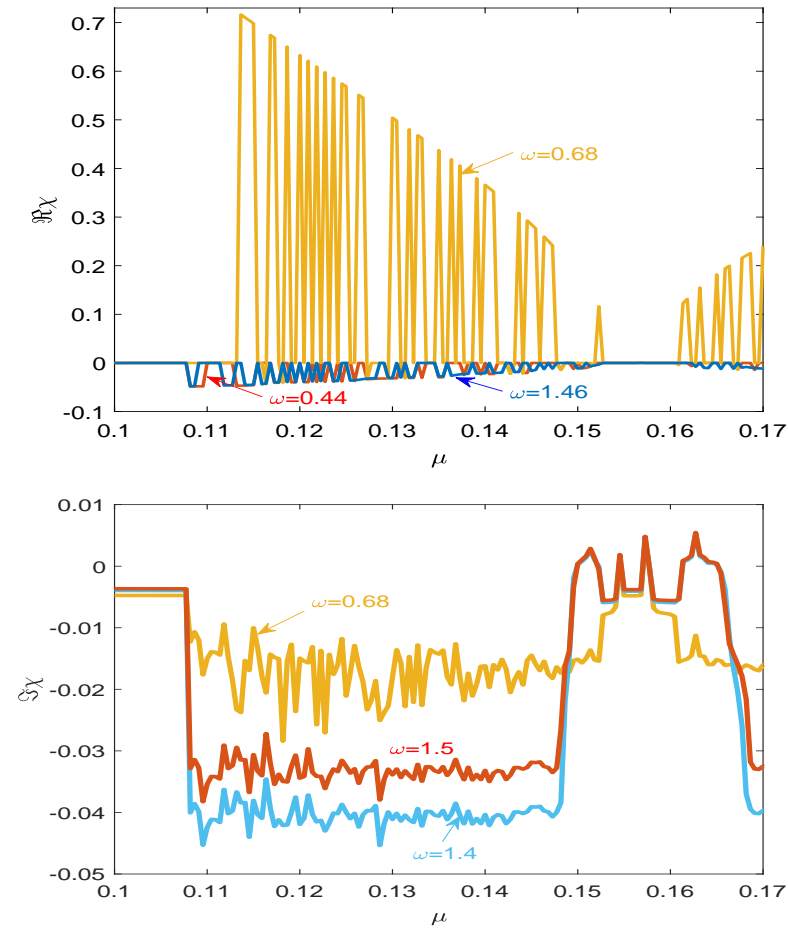

Figure 6: Color online. The steady state susceptibility $\Re \chi(\mu)$ (top panel) and $\Im \chi(\mu)$ (bottom panel) as function of electrochemical potential $\mu$. The other parameters of GQD are the same as in Fig. 4. The respective values of $\omega$ are shown in figure. Physically, the sharp switching occur when $\mu$ hits the quantized level positions, which are dense for the relatively broad stripe.

this way one eliminates certain electron-phonon scattering processes, e.g., by using a stripe-shaped active region with zigzag edges. Similarly, one excludes the indirect inter-level transitions which cause the acoustic phonon emission. The remaining contribution originates solely from the direct inter-level electron-hole recombination processes providing emission the $\mathrm{THz}$ photons out the active region. (b) Forming an optimal energy spectrum inside the active region. An increased width of the electron energy levels restricts the device performance, widens overall frequency interval and causes line broadening of the generated T-beam. One solution is to designing an active region with narrow $(\eta<<\Delta)$ quantized energy levels. It can be accomplished by placing the appropriate split gates right on the top of graphene sheet (see Fig. 2a). An example of the energy diagram of the gate/open graphene region boundary is sketched in Fig. 2b). The electric potential penetrates from the gate region into the open graphene on the Debay screening length $0.5-2 \mathrm{~nm}$, depending on the temperature and the charge carrier concentration. Figs. 22 , d show the electron subband structure and the local electron density of states in the active region respectively. (c) A distinguished feature of graphene is anisotropy of the microscopic transport. Therefore one should design the active region with 


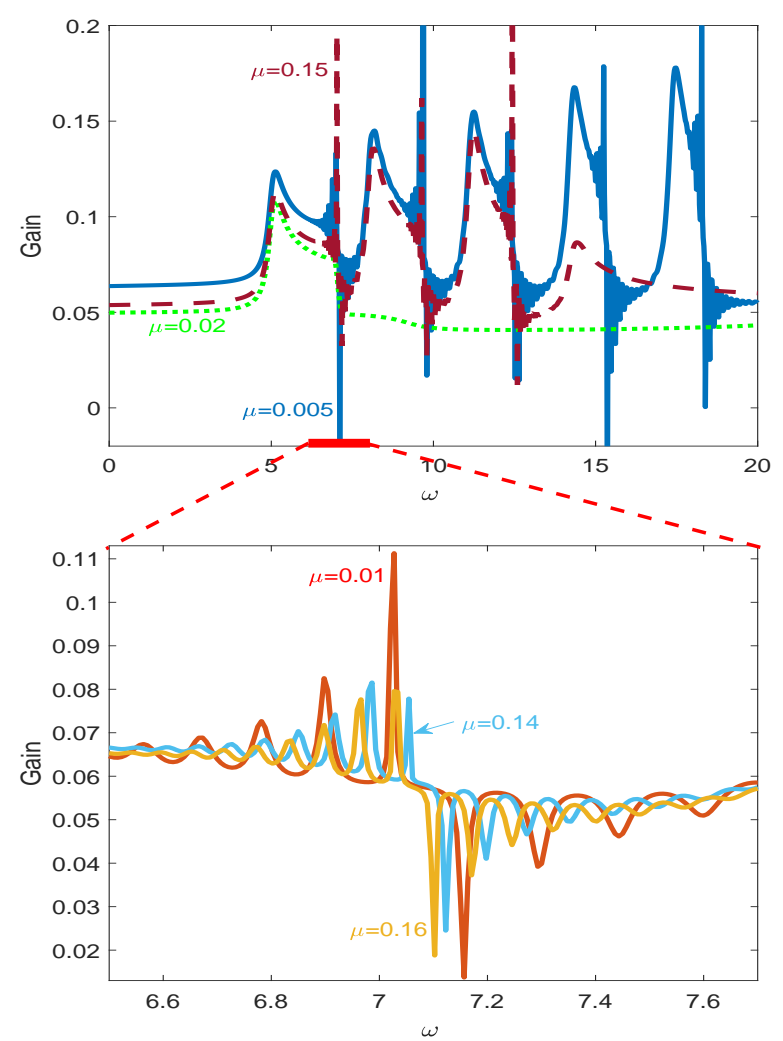

Figure 7: Color online. Top panel: Gain as function of frequency $\omega=2 \pi f$ in GQD computed for the stripe width $W=2 h v_{\mathrm{F}} / \Delta$, temperature $T=1.3 \Delta / k_{\mathrm{B}}$, the ineleastic collision rate $\eta=0.15 \Delta$, the level spacing (Stark splitting) $2 \Delta$ and the ac field amplitude $E_{\mathrm{ac}}^{(0)}=2.3 \Delta / \mathrm{eW}$. The respective values of electrochemical potential $\mu$ inside the active region are shown in figure. Bottom panel: Blowup of the frequency region denoted by red in the top panel that detalizing the fine structure of the gain function near the lowest resonant frequency.

appropriate dimensions and orientation. In this way, the major $\mathrm{THz}$ laser parameters can be well defined during the fabrication process.

There are several reasons why the graphene $\mathrm{THz}$ lasers have a remarkable potential as compared to their conventional semiconducting counterparts. (i) The intrinsic coherence in graphene is preserved far better than in other non-superconducting electronic materials. It happens due to so-called pseudospin conservation which is an intrinsic feature of graphene. In particular, the good intrinsic coherence helps to reducing the intrinsic noises. (ii) The energy relaxation in graphene is typically much slower than in other conductors. It allows achieving a considerable degree the inverse level population. (iii) Technically, the energy dependence of the election density of states in the 2D graphene enables manipulating of their properties by mere applying electric potentials to the gate electrodes. Furthermore, the 2D geometry is well suitable to fabricating the bottom, top and side gate
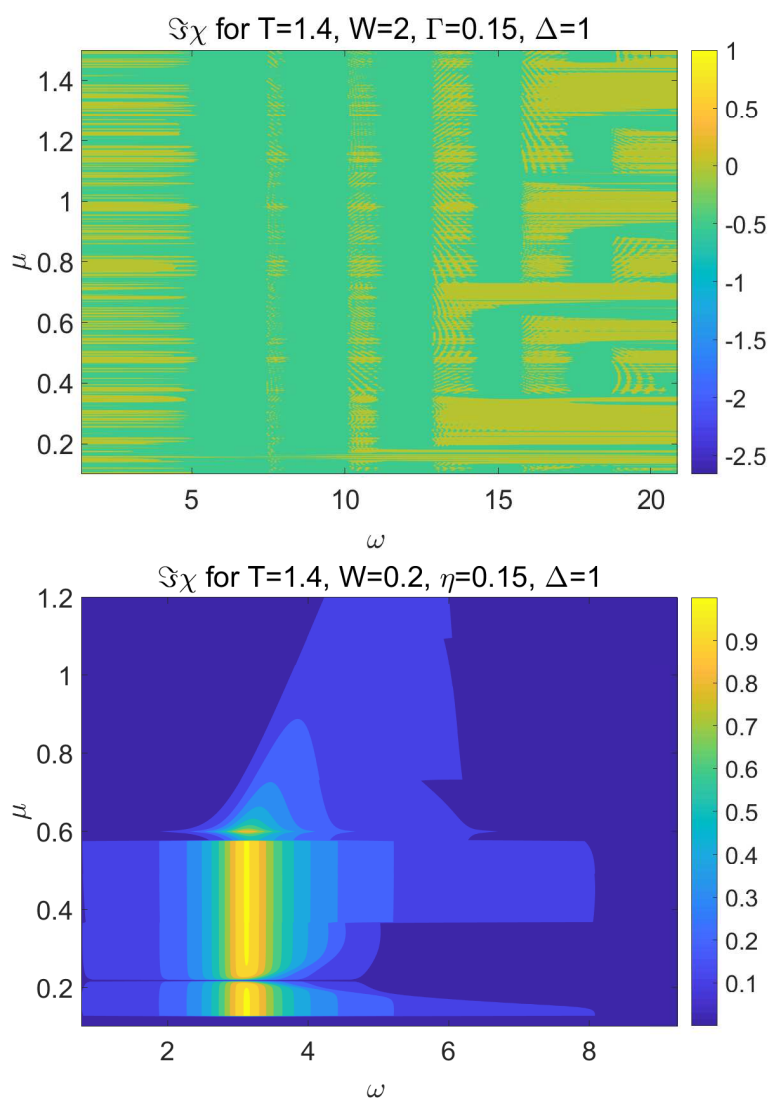

Figure 8: Color online. Top panel: Contour plot illustrating the sign switching of $\Im \chi(\mu, \omega)$ for the graphene quantum dot versus the frequency $\omega$ and electrochemical potential $\mu$ (both in units of $\Delta$ ) on the larger scale. Here the green regions correspond to $\Im \chi<0$ while yellow areas to $\Im \chi>0$. The GQD parameters are the same as in Fig. 7. Bottom panel: Contour plot $\Im \chi(\mu, \omega)$ for much narrower stripe $W=0.2 h v_{\mathrm{F}} / \Delta$. The dependence is much smoother but the sign does not change.

electrodes. Owing to $(i)$ - (iii), GQD comprises a system with the robust, voltage-controlled narrow quantized energy levels, a considerable inverse level population, good accumulation of the pumped energy, which generates a very strong $\mathrm{THz}$ monochromatic beam.

The dissipative processes inside the active region cause fluctuations that can be approximately described in terms of the quantum mechanical Langevin equations. In this way one finds that the noise terms due to spontaneous emission is $\propto r_{s p}$ and the nonradiative transition noise is $\propto r_{n r}$.

\section{NON-LINEAR ELECTROMAGNETIC PROPERTIES OF GQD}

The unconventional excitation spectrum of the chiral fermions in the graphene stripe results in non-linear electromagnetic properties of this the two-dimensional atomic monolayer material. In particular, graphene has 
remarkable non-linear properties in the terahertz $(\mathrm{THz})$ frequency range. These create many suggestions for novel photonic devices, such as $\mathrm{THz}$ devices [4], optical modulators [45], photodetectors [46] and polarizers [47]. One promising direction is exploiting the non-linear electromagnetic response of the graphene stripe to an ac electromagnetic field. Such the nonlinear effect might be used for the frequency multiplication or for self-focusing of two dimensional Townes-like solitons in the electrically tunable metastructure shown in Fig.1. Doping graphene by applying the electric potentials to the gate electrodes allows fine-tuning the nonlinear properties of such the metastructure. Total internal reflection at the boundaries of the dielectric waveguide causes the confinement of the $E$-field along the lateral $x$-direction. As the $y$ coordinate is varied along the stripe axis, the normalized $E$-field cross-section along the $x$-direction changes. Such the change, which is larger than in planar nonlinear waveguides corresponds to a significant nonlinear optical current supported by the 2D graphene stripe. Below we find that the third-order susceptibility in the graphene stripe is large enabling to form the TE and TM spatial optical solitons. Stable Townes-like spatial solitary waves propagating in the longitudinal direction originate from the intraband current dominating the electron dynamics for $\mathrm{THz}$ excitations of doped graphene. Significant magnitudes of the nonlinear optical susceptibilities in the 2D graphene sheets were theoretically predicted in Refs. [11], [12]. They have been experimentally observed for thirdorder nonlinear effects by authors of Ref. [13].
A nonlinear effect utilized to controlling light propagation at the micro- and nano-scales is the formation of temporal and spatial EM-solitons [14]. We analyze the respective non-linear contribution for the graphene strip in the geometry shown in Fig. 1. Consider a classical 2D particle with the charge $-e$ and the energy spectrum (4), (5) as for a chiral fermion in the graphene stripe with zigzag edges exposed to the time-dependent harmonic $y$-polarized electric field $E_{\mathrm{ac}}(t)=E_{\mathrm{ac}}^{0} \cos \Omega t$. The relevant excitations are electrons in the vicinity of one gap edge while taking into account the presence of two non-equivalent gap regions in the Brillouin zone by introducing the valley-degeneracy factor $g_{v}=2$. According to the Newton equation of motion

$$
\frac{d k_{y}}{d t}=-\frac{e}{\hbar} E_{\mathrm{ac}}(t)
$$

where we assume that the ac field is polarized along the stripe $y$-axis. In Eq. 22, the momentum $k_{y}(t)$ is given by

$$
k_{y}(t)=k_{0}(t)=\varepsilon \sin \Omega t,
$$

where $\varepsilon=e E_{\mathrm{ac}}^{0} / \hbar \Omega$.

In conventional $2 \mathrm{D}$ electron systems with the parabolic energy dispersion, the velocity $v_{y}$, and hence, the current $j_{y}=-e n_{s} v_{y}$ are proportional to $\hbar k_{y}$, so that the normal $2 \mathrm{D}$ system responds at the same frequency where $n_{s}$ is the areal density of change carriers. This is different for the graphene stripe where the velocity

$$
v_{y}=\frac{1}{\hbar} \frac{\partial E_{p}}{\partial k_{y}}=v_{\mathrm{F}} \frac{k_{y}}{\sqrt{k_{x}^{2}+k_{y}^{2}(t)+\left(\Delta / v_{\mathrm{F}}\right)^{2}}}=v_{\mathrm{F}} \frac{\varepsilon \sin \Omega t}{\sqrt{k_{x}^{2}+\varepsilon^{2} \sin ^{2} \Omega t+\left(\Delta / v_{\mathrm{F}}\right)^{2}}}
$$

is not merely proportional to $k_{y}$. In the extreme limit, when $k_{x}$ and $\Delta / v_{\mathrm{F}}$ in Eq. (24) are close to zero, $v_{y}$ is proportional to $\operatorname{sgn}\left(p_{x}\right)$ and the ac electric current $j_{y}=$ $-e n_{s} v_{y}$ has anharmonic contributions

$$
j_{y}(t)=e n_{s} v_{\mathrm{F}} \frac{4}{\pi}\left\{\sin \Omega t+\frac{1}{3} \sin 3 \Omega t+\frac{1}{5} \sin 5 \Omega t+\ldots\right\} .
$$

Both, the gate voltage and chemical doping can shift the chemical potential $\mu$ of electrons in graphene to the upper $E_{p 2}$ or to the lower $E_{p 1}$ band. Let us assume that the chemical potential $\mu$ lies in the upper band $E_{p 2}=$ $v_{\mathrm{F}} p$, the temperature is small, $k_{B} T<<\mu$, and the system is subjected to the time-dependent ac electric field $E_{\text {ac }}(t)$. Then the momentum distribution function of electrons $f_{p}(t)$ is described [11] by Boltzmann equation

$$
\frac{\partial f_{p}(t)}{\partial t}-\frac{\partial f_{p}(t)}{\partial \mathbf{p}} e \mathbf{E}_{\mathrm{ac}}(t)=0
$$

where we have disregarded collisions of electrons with impurities, phonons and other lattice imperfections. Equation (26) has the exact solution

$$
f_{p}(t)=\mathcal{F}_{0}\left(\mathbf{p}-\mathbf{p}_{\mathbf{0}}(\mathbf{t})\right),
$$

where

$$
\mathcal{F}_{0}(\mathbf{p})=\frac{1}{\exp \left(\frac{v_{\mathrm{F}} p-\mu}{T}\right)+1}
$$

is the Fermi-Dirac function, and

$$
\mathbf{p}_{\mathbf{0}}(\mathbf{t})=-e \int_{-\infty}^{t} d t^{\prime} \mathbf{E}_{\mathrm{ac}}\left(t^{\prime}\right)
$$

is the solution of the single particle classical equation of motion. Thus, the former equations derived in previous sections remain valid provided we replace $\mathbf{p} \rightarrow \mathbf{p}-\mathbf{p}_{\mathbf{0}}(t)$ in the respective distribution functions. 
The non-linear regime (25) is achieved at $\left|p_{0}\right| \gg p_{F}$, or at

$$
\mathcal{E} \equiv \frac{e E_{\mathrm{ac}}^{0} v_{\mathrm{F}}}{\Omega \mu}>>1
$$

According to Eq. (30), the non-linear effect becomes essential alredy at $E_{\mathrm{ac}}^{0} \geq 1.4 \mathrm{kV} / \mathrm{cm}$ provided $f=\Omega / 2 \pi=$ $1 \mathrm{THz}$ and $\mu=0.06 \mathrm{eV}$. Meaning of the above relationship (30) is that the energy, gained by electrons from the ac field during the oscillation period should be large as compared to their average equilibrium energy. In the low-field limit, the response is linear (i.e., the $j(t)$ dependence has a sinusoidal form), while at strong fields the time dependence of the current tends to that given by Eq. (25). The strong-field condition (30) can be rewritten as

$$
E_{\mathrm{ac}}^{0}>>\frac{2 \Omega \sqrt{\pi n_{s}}}{e \sqrt{g_{s} g_{v}}}
$$

which means that the required ac electric field grows linearly with the electromagnetic wave frequency and with the square root of the electron density.

There are following limitations on applicability of the quasi-classical method to describing the electromagnetic response of graphene stripe. Physically, using the Newton equation (22), one takes into account contribution the intra-band transitions to the ac electric current while ignoring the inter-band transitions between the lower quasi-hole and the upper quasi-electron bands. This is only possible if the frequency of the electromagnetic radiation satisfies the inequality

$$
\Omega \ll \max \{\mu, T\} .
$$

At room temperature and for the electric charge carrier densies $n_{s} \simeq 10^{11}-10^{12} \mathrm{~cm}^{-2}$ the above inequality limits the frequency band to $\sim 10-30 \mathrm{THz}$.

We estimate of the third-order susceptibility $\chi_{\mathrm{gr}}^{(3)}$ by computing the relevant Fourier coefficients of the timedependent $\chi\left(t, \mathbf{E}_{e x t}(t)\right)$ as

$$
\chi_{\mathrm{gr}}^{(n)}\left(\Omega, \mathbf{E}_{e x t}^{0}\right)=\frac{\Omega}{2 \pi} \int_{0}^{2 \pi / \Omega} \chi\left(t^{\prime}, \mathbf{E}_{e x t}\left(t^{\prime}\right)\right) e^{-i \Omega n t^{\prime}} d t^{\prime},
$$

where $\Omega$ is the ac field frequency, $n$ is the integer number. Likewise, we also expand the electric current density in graphene stripe $j_{\mathrm{gr}}$ in powers of $\psi=e A / p_{F}$ (where $\mathrm{A}$ is the vector potential) up to the third order:

$$
j_{\mathrm{gr}} \simeq \frac{\psi}{\sqrt{1+\psi^{2}}} \simeq \psi-\frac{\psi^{3}}{8}
$$

finding the nonlinear third order intraband current density $j_{\mathrm{gr}}^{(3)}$.

Harmonics of the ac field-dependent non-linear susceptibility $\chi_{\mathrm{gr}}^{(n)}\left(\Omega, \mathbf{E}_{\text {ext }}^{0}\right)$ are computed numerically using the above formulas. In Fig. 9 we show the Fourier components of the current versus the field parameter $\mathcal{E}$
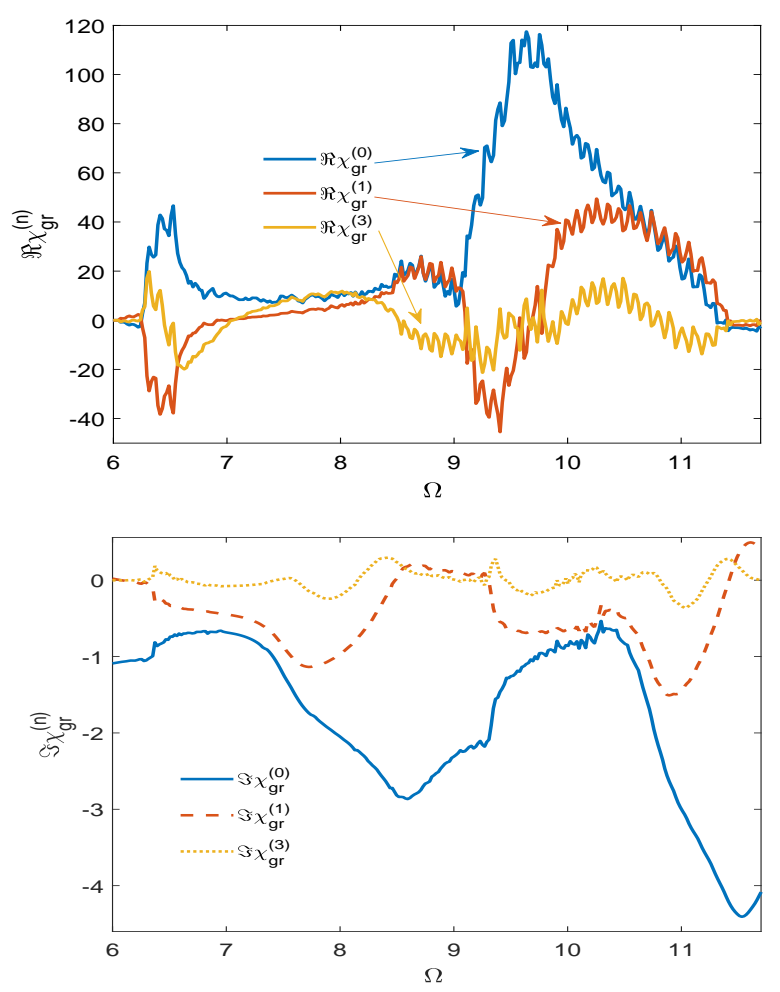

Figure 9: Color online. Top panel: The $\Re \chi_{\mathrm{gr}}^{(n)}\left(\Omega, \mathbf{E}_{\text {ext }}^{0}\right)$ for the harmonics $n=0,1,3$ as functions of the ac field frequency $\Omega$. The GQD parameters are as follows: The stripe width $W=2 h v_{\mathrm{F}} / \Delta$, temperature $T=1.2 \Delta / k_{\mathrm{B}}$, the ineleastic collision rate $\eta=0.12 \Delta$, the level spacing (Stark splitting) $2 \Delta$ and the ac field amplitude $E_{\mathrm{ac}}^{(0)}=2.3 \Delta / \mathrm{eW}$. Bottom panel: Respective plots for $\Im \chi_{\mathrm{gr}}^{(n)}\left(\Omega, \mathbf{E}_{\text {ext }}^{0}\right)$. One can see that the magnitudes of all the harmonics are comparable with each other.

given by Eq. (30). When $\mathcal{E}$ becomes larger than $\simeq 4$, the Fourier amplitudes saturate and one gets in the ultimate non-linear regime. From the plots of susceptibility harmonics $\Re \chi_{\mathrm{gr}}^{(0,1,3)}\left(\Omega, \mathbf{E}_{\text {ext }}^{0}\right)$ and $\Im \chi_{\mathrm{gr}}^{(0,1,3)}\left(\Omega, \mathbf{E}_{\text {ext }}^{0}\right)$ illustrating the non-linear effects in graphene stripe with zigzag edges shown in Fig. 9 one can see that the 0 -th, 1st and 3-harmonics of $\chi\left(t^{\prime}, \mathbf{E}_{\text {ext }}\left(t^{\prime}\right)\right)$ are about the same order of magnitude, which suggests the significance of non-linear phenomenon in the GQD system under consideration.

In conclusion of this Section, due to the unconventional dispersion law (4), (5) of the chiral fermions, the response of graphene stripe to an ac electromagnetic field is intrinsically non-linear.

\section{TIME EVOLUTION OF SUSCEPTIBILITY}

An accurate estimation of the time required to reach the steady state regime represents a tedious task involving a self-consistent solution of a complex system of the 


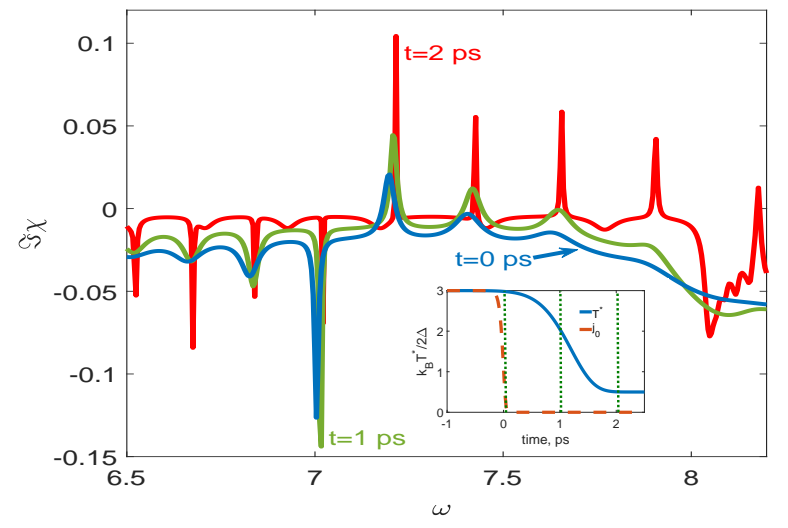

Figure 10: Color online. The imaginary part of optical susceptibility $\Im \chi(\omega, t)$ as functions of the frequency $\omega$ at time moments $t=0 \mathrm{ps}, 1 \mathrm{ps}$ and 2 ps after the injection current pulse ends. Here we used the GQD stripe width $W=2 h v_{\mathrm{F}} / \Delta$, the steady state temperature $T=0.5 \Delta / k_{\mathrm{B}}$, the level spacing (Stark splitting) $2 \Delta$ and the energy relaxation time $\tau_{\mathrm{HF}}=1 \mathrm{ps}$. One can see that the steady state of GQD is achieved at $t \sim 2 \mathrm{ps}$, after the injection current pulse ends and the HF excitations recombine in the energy. Inset shows the time dependence of the effective temperature $T^{*}(t)$ (solid blue line) that achieves its steady state value $T=0.5 \Delta / k_{B}$ at $t \sim 2 \mathrm{ps}$, after the injection current pulse (red dash) ends at $t=0$.

Boltzmann equations complemented by equations for the HF excitation spectrum. Besides, the equations must be complemented by respective boundary conditions defining the geometry and initial state. In this work we provide just a simplest insight how the optical susceptibility $\chi^{\prime \prime}(\omega, t)$ evolves in response to a pulse of injection current incurring a sharp change of the effective electron temperature $T^{*}$ of GQD. Let us assume that the pulse of injection current heats GQD, whose effective temperature increases up to $T^{*}$. Basically, after the pulse ends, the time evolution of the HF distribution function $f(\epsilon, t)$ is found as a solution of Boltzmann equation. For the sake of simplicity, we use the effective temperature approximation that gives

$$
f(\epsilon, t)=\frac{1}{\exp \left(\frac{\epsilon-\mu}{T^{*}(t)}\right)+1},
$$

where the time dependence of $T^{*}$ is determined by the energy relaxation of the HF distribution function. In the above approximation we use

$$
T^{*}(t)=T \exp \left[\left(-\frac{t-t_{0}}{\tau_{p}}\right)^{n}\right],
$$

where we take $n=100$ and $t_{0}=\tau_{p}=2.5 \mathrm{~ns}$. When the energy recombination time $\tau_{\mathrm{HF}}$ of the HF excitations is very short, $\tau_{\mathrm{HF}}<<\min \left\{\tau_{p}, t_{0}\right\}$ (typically $\tau_{\mathrm{HF}} \sim$ $10^{-12} \mathrm{~s}$ ), the time evolution of $T^{*}(t)$ immediately follows the change of injection current, $T^{*}(t) \propto j(t)$. However, the scenario becomes different when $\tau_{\mathrm{HF}} \sim\left\{\tau_{p}, t_{0}\right\}$. In the last case, when the injection current pulses are sufficiently short, the optical susceptibility reaches the steady state on the timescale $\sim \tau_{\mathrm{HF}}$. Such the time evolution of $\Im \chi(\omega, t)$ is illustrated in Fig. 10 by using the above simple model (35) and (36) allowing to determine the behavior of the graphene "particle" from the time that the injection current pulse ends $(t=0 \mathrm{ps})$ till reaching steady state at $t=2 \mathrm{ps}$. The graph in Fig. 10 helps to understand and evaluate the role of non-equlibrium effect in GQD.

\section{CONCLUSIONS}

Obtained results suggest that the considered design of the graphene quantum dot (GQD) allows the allelectrical control of the optical susceptibility $\chi(\omega, \mu)$. This becomes possible because the magnitude and sign of both the real and imaginary parts of $\chi(\omega, \mu)$ depend on the electrochemical potential $\mu$ and on the frequency variable $\omega$. Technically, in the GQD structure one can change the magnitudes of $\Delta$ and $\mu$ by applying appropriate electric potentials to the local gates as depicted in Fig. 1, thereby enabling the flexible control of the GQD optical properties.

Furthermore, likewise the pristine graphene, the GQD structure has remarkable non-linear electromagnetic properties stemming from the unconventional dispersion law of the chiral fermions in the graphene stripe with zigzag edges. A strong non-linear effect arises because the optical susceptibility depends on the ac field intensity. The magnitude of high-order harmonics is significant even in relatively weak ac fields, causing appearance of variety the non-linear effects.

In experiments, the $\mathrm{THz}$ radiation is detected in several ways. For instance, one can form a Josephson junction in an adjacent area on the same substrate, which will serve as a $\mathrm{THz}$ detector. Another option is to deposit GQD THz detector on the same substrate next to the GQD THz emitter. Furthermore, one can use metallic co-planar strip lines as $\mathrm{THz}$ antennas to detect the T-rays. Special attention must be paid to creating of the sharp and narrow electron quantized levels formed between two timber-like multilayered gate electrodes deposited along the ZZ-direction. Such the energy levels are robust in presence of lattice defects and imperfections remaining to be very narrow and sharp since they are topologically protected $[4,32, \underline{36}]$. In the active region of the $\mathrm{THz}$ emitter, the coherent monochromatic $\mathrm{THz}$ waves originate from the quantum transitions between the sharp localized levels. The energy level splitting is readily controlled by the voltage difference between the gate electrodes having a multilayered structure as depicted in Fig. 1.

The unconventional electromagnetic (EM) properties of the graphene quantum dots (GQD) have a promising potential for practical applications. Experimentally, 
it would be interesting to fabricating the all-electrically controlled GQD based on the graphene stripes with atomic zigzag edges. The calculation results of the optical susceptibility indicate strong dependence on the frequency and electrochemical potential, which can be exploited for experimental observing the tunable THz emission. Another result is the non-linear electromagnetic response of GQD, whose mechanism is related to the unconventional properties of chiral fermions in graphene stripes with atomic zigzag edges. The obtained data allow better understanding the physical mechanisms related to the electrically controlled GQD showing remarkable electromagnetic properties.
[1] X. Liu, Z. Chen, E. P. J. Parrott, B. S. Y. Ung, J. Xu, E. PickwellMacPherson, Graphene Based Terahertz Light Modulator in Total Internal Reflection Geometry, Advanced Optical Materials, 5, 1600697 (2017).

[2] Z. Chen, X. Chen, L. Tao, K. Chen, M. Long, X. Liu, K. Yan, R. I. Stantchev, E. PickwellMacPherson, J.B. Xu, Graphene controlled Brewster angle device for ultra broadband terahertz modulation, Nature Communications, 9, 4909 (2018).

[3] T. Low, A. Chaves, J. D. Caldwell, A. Kumar, N. X. Fang, P. Avouris, T. F. Heinz, F. Guinea, L. Martin-Moreno, F. Koppens, "Polaritons in layered two-dimensional materials," Nat. Mater. 16, 182a, 194 (2017).

[4] S. Shafraniuk, Thermoelectricity and Heat Transport in Graphene and Other 2D Nanomaterials (Elsevier - Health Sciences Division, 2016), ISBN-13: 9780323443975.

[5] S. E. M. Rinzan, G. Jenkins, H. D. Drew, S. Shafranjuk, and P. Barbara, Carbon Nanotube Quantum Dots As Highly Sensitive Terahertz-Cooled Spectrometers, Nano Lett., 12(6), 3097-3100 (2012).

[6] T. Fuse, Y. Kawano, T. Yamaguchi, Y. Aoyagi, K. Ishibashi, Quantum response of carbon nanotube quantum dots to terahertz wave irradiation, Nanotechnology, 18, 044001 (2007).

[7] S. Chakraborty, O. P. Marshall, T. G. Folland, Y. -J. Kim, A. N. Grigorenko, K. S. Novoselov, Gain modulation by graphene plasmons in aperiodic lattice lasers, Science 351, 6270, 246-252 (2016). DOI: 10.1126/science.aad2930.

[8] D. Bimberg, M. Grundmann, and N. N. Ledentsov, Quantum Dot Heterostructures Wiley, New York, 1999 .

[9] D. Bimberg, M. Kuntz, and M. Lämmlin, Appl. Phys. A 80, 11792005 .

[10] D. Bimberg, Electron. Lett. 44, 1682008 .

[11] S. A. Mikhailov, Non-linear electromagnetic response of graphene, Europhys. Lett. 79, 27002 (2007).

[12] K. L. Ishikawa, Nonlinear optical response of graphene in time domain, Phys. Rev. B 82, 201402(R) (2010).

[13] E. Hendry, P. Hale, J. Moger, A. Savchenko, and S. Mikhailov, Coherent Nonlinear Optical Response of Graphene, Phys. Rev. Lett. 105, 097401 (2010).

[14] R. W. Boyd, Nonlinear Optics (Academic Press, New York, 1992), ISBN: 9780123694706.

[15] L. Ju, B. Geng, J. Horn, C.Girit, M. Martin, Z. Hao, H. A. Bechtel, X. Liang, A. Zettl, Y. Ron Shen, and F. Wang, Graphene plasmonics for tunable terahertz metamaterials, Nat. Nanotech. 6, 630-634 (2011).

[16] T. Eberlein, U. Bangert, R. R. Nair, R. Jones, M. Gass, A. L. Bleloch, K. S. Novoselov, A. Geim, and P. R. Briddon, Plasmon spectroscopy of free-standing graphene films, Phys. Rev. B 77, 233406 (2008).

[17] J. T. Kim, and S.-Y. Choi, "Graphene-based plasmonic waveguides for photonic integrated circuits," Opt. Express vol. 19, pp. 24557-24562, 2011.

[18] J. Chen , M. Badioli, P. Alonso-González, S. Thongrattanasiri, F Huth, J. Osmond, M. Spasenović, A. Centeno, A. Pesquera, P. Godignon, A. Z. Elorza, N. Camara, F. J. G. de Abajo, R. Hillenbrand, F. H. Koppens, "Optical nano-imaging of gate-tunable graphene plasmons," Nature vol. 487, pp. 77-81, 2012, doi: 10.1038/nature11254.

[19] Z. Fei, A. S. Rodin, G. O. Andreev, W. Bao, A. S. McLeod, M. Wagner, L. M. Zhang, Z. Zhao, M. Thiemens, G. Dominguez, M. M. Fogler, A. H. Castro Neto, C. N. Lau, F. Keilmann \& D. N. Basov, "Gatetuning of graphene plasmons revealed by infrared nanoimaging," Nature, vol. 487, pp. 82-85, 2012.

[20] A. N. Grigorenko, M. Polini, and K. S. Novoselov, "Graphene plasmonics," Nat. Photon. vol. 6, pp. 749$758,2012$.

[21] H. Yan et al., "Damping pathways of mid-infrared plasmons in graphene nanostructures," Nat. Photon. vol. 7, pp. 394-399, 2013.

[22] X. Wang, Z. Cheng, K. Xu, H. K. Tsang, and J.-B. Xu, "High- responsivity graphene/silicon-heterostructure waveguide photodetectors," Nat. Photon. vol. 7, pp. 888-891, 2013.

[23] E. O. Polat and C. Kocabas, "Broadband Optical Modulators Based on Graphene Supercapacitors," Nano Lett. vol. 13, pp. 5851-5857, 2013.

[24] K. S. Novoselov et al., "A roadmap for graphene," Nature, vol. 490, pp. 192-200, 2012.

[25] W. L. Barnes, A. Dereux, and T. W. Ebbesen, "Surface plasmon subwavelength optics," Nature 424, pp. 824-830, 2008.

[26] Yu.V. Bludov, D.A. Smirnova, Yu.S. Kivshar, N.M.R. Peres, and M.I. Vasilevskiy,"Nonlinear TE-polarized surface polaritons on graphene," Phys. Rev. B, vol. 89, pp. 035406-035412, 2014.

[27] E. Ozbay, Plasmonics: Merging Photonics and Electronics at Nanoscale Dimensions, Science 311, 189-193 (2006).

[28] C. J. Docherty, C.-T. Lin, H. J. Joyce, R. J. Nicholas, L. M. Herz, L.-J. Li, M. B. Johnston, Extreme sensitivity of graphene photoconductivity to environmental gases, Nat. Commun. 3, 1228-1235 (2012).

[29] A. Das, S. Pisana , B. Chakraborty, S. Piscanec, S. K. Saha, U. V. Waghmare, K. S. Novoselov, H. R. Krishnamurthy, A. K. Geim, A. C. Ferrari, A. K. Sood, Monitoring dopants by Raman scattering in an electrochemically top-gated graphene transistor, Nat. Nanotech. 3, 210-215 (2008).

[30] F. Bonaccorso, Z. Sun, T. Hasan, and A. C. Ferrari, 
Graphene photonics and optoelectronics, Nat. Photon. 4, 611-622 (2010).

[31] A. Tredicucci and M. S. Vitiello, Device concepts for graphene-based terahertz photonics, IEEE J. Sel. Top. Quantum Electron., 20, 130-138 (2014).

[32] S. Shafranjuk, Graphene: Fundamentals, Devices, and Applications, (Pan Stanford, 2015), SBN 9789814613477 - CAT\# N11214, 634.

[33] P. Ruffieux, S. Wang, B. Yang, C. Sánchez-Sánchez, J. Liu, T. Dienel, L. Talirz, P. Shinde, C. A. Pignedoli, D. Passerone, T. Dumslaff, X. Feng, K. Müllen, R. Fasel, On-surface synthesis of graphene nanoribbons with zigzag edge topology, Nature 531, 489-492 (2016).

[34] L. Brey, H.A. Fertig, Edge states and the quantized Hall effect in graphene, Phys. Rev. B 73 (19), 195408-195416 (2006).

[35] L. Brey, H.A. Fertig, Electronic states of graphene nanoribbons studied with the Dirac equation, Phys. Rev. B, 73 (23), 235411-235419 (2006).

[36] Hossain, M.S., Al-Dirini, F., Hossain, F.M., Skafidas, E., High performance graphene nano-ribbon thermoelectric devices by incorporation and dimensional tuning of nanopores. Sci. Rep. 5, 11297-11309 (2015).

[37] S. E. Shafranjuk, Electromagnetic properties of graphene junctions, European Physical Journal, B 80, 379-393 (2011).

[38] E. Yablonovich, Inhibited Spontaneous Emission in Solid-State Physics and Electronics, Phys. Rev. Lett. 58, 2059-2062 (1987).

[39] T. Pickering, J. M. Hamm, A. F. Page, S. Wuestner, and O. Hess, Cavity-free plasmonic nanolasing enabled by dispersionless stopped light, Nat. Commun. 5, 49724978 (2014).

[40] J. P. Dowling, M. Scalora, M. J. Bloemer, and C. M. Bowden, The photonic band edge laser: A new approach to gain enhancement, J. Appl. Phys. 75, 1896-1906 (1994).

[41] M. Engel, M. Steiner, A. Lombardo, A. C. Ferrari, H. v. Löhneysen, P. Avouris, R. Krupke, Light-matter interaction in a microcavity-controlled graphene transistor, Nat. Commun. 3, 906-912 (2012).

[42] J. Li, M. Jiang, C. Xu, Y. Wang, Y. Lin, J. Lu, Z. Shi, Plasmon coupled Fabry-Perot lasing enhancement in graphene/ZnO hybrid microcavity, Sci. Rep. 5, 92639263 (2015).

[43] A. H. Castro Neto, F. Guinea, N. M. R. Peres, K. S. Novoselov, and A. K. Geim, The electronic properties of graphene, Rev. Mod. Phys. 81, 109 (2009).

[44] D. Sun, C. Divin, J. Rioux, J. E. Sipe, C. Berger, W. A. de Heer, P. N. First and Th. B. Norris, Coherent Control of Ballistic Photocurrents in Multilayer Epitaxial Graphene Using Quantum Interference, Nano Lett., 10 (4), 1293â 1296 (2010) DOI: 10.1021/nl9040737

[45] M. Liu, X. Yin, E. Ulin-Avila, B. Geng, Th. Zentgraf, L.
Ju, F. Wang \& X. Zhang, A graphene-based broadband optical modulator, Nature 474, 64 (2011).

[46] T. Mueller, F. N. Xia and P. Avouris, Graphene photodetectors for high-speed optical communications, Nature Photonics 4, 297 (2010).

[47] Q. Bao, H. Zhang, B. Wang, Zh. Ni, C. Haley Y. X. Lim, Y. Wang, D. Y. Tang \& K. P. Loh, Broadband graphene polarizer, Nature Photonics 5, 411 (2011).

[48] M. L. Nesterov, J. Bravo-Abad, A. Yu. Nikitin, F. J. Garcia-Vidal, and L. Martin-Moreno, "Graphene supports the propagation of subwavelength optical solitons", Laser Photonics Rev. 7, No. 2, L7â, L11 (2013) / DOI 10.1002/lpor.201200079.

[49] S. Shafraniuk, Another approach to the problem of room temperature superconductivity, Quantum Stud.: Math. Found., Springer Nature, DOI 10.1007/s40509-017-01359 (2017).

[50] D. Rodrigo, T. Low, D. B. Farmer, H. Altug, and P. Avouris, Plasmon coupling in extended structures: Graphene superlattice nanoribbon arrays, Phys. Rev. B93, 125407 (2016).

[51] M. Mattheakis, C. A. Valagiannopoulos, and E. Kaxiras, Epsilon-near-zero behavior from plasmonic Dirac point: Theory and realization using two-dimensional materials Phys. Rev. B94, 201404(R) (2016).

[52] I. S. Nefedov, C. A. Valagiannopoulos and L. A. Melnikov, Perfect absorption in graphene multilayers, Journal of Optics, 15, 11 (2013).

\section{APPENDIX}

\section{A. Susceptibility of pristine graphene}

Here we provide technical details concerning the optical susceptibilty of pristine graphene. We use Eq. (1), which is written for indirect interband transitions accompanied by the absorption/emission of a phonon with the finite momentum $\mathbf{q} \neq \mathbf{0}$. Here we use units with $\hbar=1$ and $k_{\mathrm{B}}=1$ if not stated otherwise. When the absorption/emission process is accompanied by the photon instead of the phonon, the interband transitions become direct and the above Eq. (1) is simplified. For the photons, whose absorption creates the electron-like HF (below for brevity we call them electrons) in the conductive band while the hole-like HF (we call them holes) in the valence band (direct interband transitions), we set $\mathbf{q}=\mathbf{0}$. Then, using that $s s^{\prime}=-1$ we get the respective energy change as $\epsilon_{s^{\prime}, v}-\epsilon_{s, c}= \pm 2 \epsilon$ and from Eq. (1) we get

$$
\begin{aligned}
\chi(\omega)= & -\frac{6 \sqrt{3}\left|d_{c v}\right|^{2}}{\pi v_{F}^{2}} \int_{0}^{v_{F} \Lambda} d \epsilon \epsilon(f(-\epsilon)-f(\epsilon)) \\
& \times\left(\frac{1}{\omega+2 \epsilon+i \eta}-\frac{1}{\omega-2 \epsilon+i \eta}\right),
\end{aligned}
$$

where $\Lambda \approx 1 / a$ is the cutoff momentum and $a$ is the lattice constant. 
In beginning we find how the steady state functions $\chi(\omega)$ and $\chi(\mu)$ depend respectively on the photon frequency $\omega$ and the HF electrochemical potential $\mu$. In the above formulas we set

$$
f(\epsilon)=\frac{1}{e^{(\epsilon-\mu) / T}+1} .
$$

In the zero-temperature limit $T=0$ we simply set $f(\epsilon)=\theta(\mu-\epsilon)$ and $f(-\epsilon)=1$ for electron doping with $\mu>0$, where $\mu$ is the electron electrochemical potential. Respectively, for the hole doping we get $\mu<0$. Then

$$
\chi(\omega)=-\frac{6 \sqrt{3}\left|d_{c v}\right|^{2}}{\pi v_{F}^{2}} \int_{\mu}^{v_{F} \Lambda}\left(\frac{1}{\omega+2 \epsilon+i \eta}-\frac{1}{\omega-2 \epsilon+i \eta}\right) \epsilon d \epsilon,
$$

where $\zeta^{2}=\left(\eta^{2}-2 i \eta \omega-\omega^{2}\right) / 4$ and we have used

$$
f(-\epsilon)-f(\epsilon)=1-\theta(\mu-\epsilon)=\left\{\begin{array}{c}
0 \text { for } \epsilon<\mu \\
1 \text { for } \epsilon>\mu
\end{array} \mid .\right.
$$

An immediate integration of Eq. (40) gives a simple analytical expression in the form

$$
\chi(\omega, \mu)=-\frac{i}{\pi} \frac{3 \sqrt{3}\left|d_{c v}\right|^{2}}{2 \pi v_{F}^{2}}\left[\Lambda-\mu+\frac{\omega+i \eta}{2}\left(\tanh ^{-1}\left(\frac{2 \mu}{\omega+i \eta}\right)-\tanh ^{-1}\left(\frac{2 \Lambda}{\omega+i \eta}\right)\right)\right] .
$$

The above calculations are illustrated in Figs. 3 where we show the real $\chi^{\prime}=\Re \chi$ and imaginary $\chi^{\prime \prime}=\Im \chi$ parts of the optical susceptibility versus the frequency $\omega$ and electrochemical potential $\mu$.

$$
\chi(\omega, \mu)=-\frac{6 \sqrt{3}\left|d_{c v}\right|^{2}}{\pi v_{F}^{2}}\left[v_{F} \Lambda-\mu+\frac{\omega}{4}\left(\ln \left|\frac{\omega+2 \mu}{\omega-2 \mu}\right|+i \pi \theta(\omega-2 \mu)\right)\right] .
$$

It is also instructive to find the number of excitations $n(\mu)$ in the $2 \mathrm{D}$ graphene. For the equilibrium case we find

$$
n=\frac{N}{L^{2}}=-\frac{12 \sqrt{3}}{\pi v_{F}^{2}} \int_{0}^{v_{F} \Lambda} \epsilon f(\epsilon) d \epsilon=-\frac{12 \sqrt{3}}{\pi v_{F}^{2}} \int_{0}^{v_{F} \Lambda} \epsilon \frac{1}{e^{\beta(\epsilon-\mu)}+1} d \epsilon-\frac{12 \sqrt{3}}{\pi v_{F}^{2} \beta^{2}} \int_{0}^{\beta v_{F} \Lambda} \epsilon \frac{1}{e^{(\epsilon-\beta \mu)}+1} d \epsilon .
$$

Then one gets

$$
n(\mu)=-\left(12 \sqrt{3} T^{2} / \pi v_{F}^{2}\right)\left[L i_{2}\left(-e^{-\frac{\mu}{T}}\right)-L i_{2}\left(-e^{\frac{\Lambda-\mu}{T}}\right)+\Lambda\left(\Lambda-2 T \log \left(e^{\frac{\Lambda-\mu}{T}}+1\right)\right) / T^{2}\right] .
$$

In more realistic conditions, e.g., when the temperature is finite while the graphene sheet is deposited on a substrate and its electronic states are controlled by gate electrodes, one finds the optical susceptibility numerically, as described in the main text. Likewise, numeric solutions also used for studying the non-stationary and nonequilibrium properties of the graphene samples. 